OPEN

SUBJECT AREAS:

ECOPHYSIOLOGY

PLANT SIGNALLING

ECOLOGY

PLANT SCIENCES

Received

7 March 2013

Accepted

18 November 2013

Published

5 December 2013

Correspondence and requests for materials should be addressed to

I.F. (i.filella@creaf. vab.cat)

\section{Floral advertisement scent in a changing plant-pollinators market}

\author{
Iolanda Filella ${ }^{1,2}$, Clara Primante ${ }^{2}$, Joan Llusià̀ ${ }^{1,2}$, Ana M. Martín González ${ }^{2,3,4}$, Roger Seco ${ }^{1,2,5}$, \\ Gerard Farré-Armengol ${ }^{1,2}$, Anselm Rodrigo ${ }^{2,6}$, Jordi Bosch ${ }^{2} \&$ Josep Peñuelas ${ }^{1,2}$
}

${ }^{1}$ CSIC, Global Ecology Unit CREAF-CEAB-UAB, Cerdanyola del Vallès, 08193 Barcelona, Catalonia, Spain, ${ }^{2}$ CREAF, Cerdanyola del Vallès, 08193 Barcelona, Catalonia, Spain, ${ }^{3}$ Pacific Ecoinformatics and Computational Ecology Lab, 1604 McGee Avenue Berkeley, CA 94703, USA, ${ }^{4}$ Center of Macroecology, Evolution and Climate, Dept. of Biology, University of Copenhagen, 15 Universitetsparken, DK-2 100, Denmark, ${ }^{5}$ Atmospheric Chemistry Division, National Center for Atmospheric Research, Boulder, CO 80301, USA, ${ }^{6}$ Univ Autònoma Barcelona, Cerdanyola del Vallès 08193, Spain.

Plant-pollinator systems may be considered as biological markets in which pollinators choose between different flowers that advertise their nectar/pollen rewards. Although expected to play a major role in structuring plant-pollinator interactions, community-wide patterns of flower scent signals remain largely unexplored. Here we show for the first time that scent advertisement is higher in plant species that bloom early in the flowering period when pollinators are scarce relative to flowers than in species blooming later in the season when there is a surplus of pollinators relative to flowers. We also show that less abundant flowering species that may compete with dominant species for pollinator visitation early in the flowering period emit much higher proportions of the generalist attractant $\beta$-ocimene. Overall, we provide a first community-wide description of the key role of seasonal dynamics of plant-specific flower scent emissions, and reveal the coexistence of contrasting plant signaling strategies in a plant-pollinator market.

M any plants produce rewards in the form of nectar and pollen that attract pollinators, thus ensuring the transfer of pollen from flower to flower. Plant-pollinator communities may thus be considered as biological markets in which pollinators choose between different flowers that may compete for their visits $^{1-3}$. Flowers rely on sensory signals to advertise their rewards, color and scent being the most important ones ${ }^{4}$. Historically, plant-pollinator relationships have mostly been considered as a visually-mediated process, and floral odors have received less consideration ${ }^{5}$. However, olfactory cues are often the basis upon which pollinators make flower choices, because scent cues are easily learned and remembered by pollinators ${ }^{6}$. Different studies have revealed that bees are able to detect pollen and nectar in flowers via odour cues $\left({ }^{6-10}\right.$, and references therein), that bees learn odours faster and remember them for longer than visual cues $^{6,11}$, that specific pollen odour plays a key role in host recognition by oligolectic solitary bees, ${ }^{7,8}$, and that floral odour differences are important for maintaining reproductive isolation between closely related plant species ${ }^{12}$. Other studies have revealed that plant and floral scents elicit a foraging response also in other insect pollinators ${ }^{13-15}$. In addition, floral scent has been found to improve plant fitness via increased pollinator attraction ${ }^{16}$. Nevertheless, in spite of the putative importance of flower odors in structuring plant-pollinator interactions, community-wide patterns of flower scent signals and their seasonal dynamics remain largely undescribed.

As in most markets, supply and demand in plant-pollinator systems fluctuate in time. Certain periods are characterized by a surplus of flowers relative to pollinators, which may result in competition between flowers and large investment in rewards and display ${ }^{1}$. Conversely, in periods exhibiting a surplus of pollinators relative to flowers, a reduction of investment in floral rewards and display is expected. In the Mediterranean region, the peak of flowering occurs in the early spring (March-April) and hot, dry summers present a physiological challenge to plants ${ }^{17-19}$. The early flowering peak results in a surplus of flowers relative to pollinators in spring, followed by a surplus of pollinators in relation to flower availability in summer ${ }^{1}$.

We studied a plant-pollinator community in a Mediterranean shrubland, in which flower and pollinator availability follow closely this model of a seasonal floral market ${ }^{20}$. To explore the existence of contrasting plant signaling strategies, we quantified floral scent compounds for each plant species as well as the seasonal variation of flower abundance, nectar and pollen availability, and flower visitation rates. Specifically, we examined two hypotheses associated with the emergence of differentiated plant-signaling strategies in plant-pollinator networks. Firstly, we hypothesized a greater investment in scent advertisement early rather than late in the flowering 
period associated with the lower pollinator availability (pollinator abundance hypothesis). Secondly, we examined whether less abundant flowering species, which, other factors being equal, might have difficulty attracting pollinators, produce a different scent from abundant species (plant abundance hypothesis). We tested these two hypotheses and provide a first integrative description of community-wide patterns of flower scent signals.

\section{Results}

The overall flowering period extended from late February to June. The community presented a clear seasonal pattern with two contrasting scenarios. Early in the flowering period, from late February until early April, flower and floral reward availability (nectar and pollen) was high and visitation rates (pollinator visits per flower and unit time) low. On the other hand, from mid April until June, flower and floral reward availability were much lower, and pollinator visitation rates were much higher (Fig. 1). Plant species were therefore divided in two groups (early and late flowering species) using as a criterion the time when the drastic decline in flower availability coincided with a drastic increase in pollinator visitation (Fig. 1). The species with their peak of flowering early in the season (flowering from late February to early April) were Rosmarinus officinalis, Thymus vulgaris (hermaphrodite and female morphs), Muscari neglectum, Ranunculus gramineus, Euphorbia flavicoma and Iris lutescens. The species with their peak of flowering in the second half (flowering from early April to June) were Cistus salvifolius, Dorycnium hirsutum, Cistus albidus, Orobanche latisquama, Gladiolus illyricus, Galium aparine, Scorpiurus muricatus, Anagallis arvensis, Convolvulus althaeoides, Centaurea linifolia, Centaurea paniculata, Sideritis hirsuta, Phlomis lychnitis, Linum strictum, Leuzea conifera and Allium sphaerocephalon. One species, Biscutella laevigata, was in bloom during most of the flowering season (March to June) and was not included in the analyses. Bees were the main flower visitors until the end of may (see Supplementary Fig. S1 online).

The floral scent of species flowering early in the season significantly differed from the floral scent of species flowering later (pseudo- $\mathrm{F}_{1,22}=8.06, \mathrm{P}<0.001$, PERMANOVA, see Supplementary Table S1 and Fig. S2 online). Species flowering early emitted higher amounts of terpenes per flower and per dry weight of flower $\left(\mathrm{F}_{1,21}=12.8 \mathrm{P}<0.01, \mathrm{~F}_{1,21}=6.03, \mathrm{P}<0.05\right.$, respectively, $\mathrm{n}=23$ species; Fig. 2a). After correcting emissions by field temperatures, species flowering early still emitted higher amounts of terpenes (per flower and per dry weight of flower $)\left(\mathrm{F}_{1,21}=9.23 \mathrm{P}<0.01, \mathrm{~F}_{1,21}=\right.$ $5.15 \mathrm{P}<0.05$, respectively, $\mathrm{n}=23$ species). These species also emitted a higher proportion of terpenes relative to total volatiles $\left(\mathrm{F}_{1,21}=\right.$ 34.2, $\mathrm{p}<0.0001$, ANOVA, Fig. 2b). There was not any significant phylogenetic signal in these differences for terpene emissions $(\mathrm{p}=$ 0.80) (see supplementary Fig. S3 online).

Among the species flowering early in the flowering period, Rosmarinus officinalis and Thymus vulgaris largely out-numbered the rest of species in number of individuals and number of flowers per individual (Fig. 3). These two species accounted by far for most of the nectar and pollen produced during this period ${ }^{20}$. Their scent was different from that of less abundant co-flowering species (pseudo$\left.\mathrm{F}_{1,6}=12.27, \mathrm{P}=0.001\right)$. Notably, these less abundant species coflowering with $R$. officinalis and T. vulgaris emitted a similar flower fragrance with a very high proportion of the monoterpene $\beta$-ocimene (Fig. 3). The percentage of $\beta$-ocimene emissions after controlling for phylogenetic relatedness was still higher in the less abundant species than in $R$. officinalis and T. vulgaris ( $\mathrm{p}=0.019, \mathrm{n}=5$, PGLS, phylogenetic generalized least square regressions). Early flowering species shared pollinators (see Supplementary Fig. S1b online), most of which were generalists in their flower-visiting habitats (see Supplementary Fig. S1 online).

No significant seasonal trend was found in the emission rates of total terpenes, nor in the emission rates of $\beta$-ocimene in particular, in any of the five species studied throughout their entire blooming period ( $R$. officinalis, E. flavicoma, M. neglectum- flowering mainly early in the season-, B. laevigata -flowering the whole season- and $P$. lychnitis-flowering late in the season) (Fig. 4).

\section{Discussion}

In accordance with the pollinator abundance hypothesis, we found that species flowering early in the season presented a higher scent emission than plant species flowering later, with the former emitting a higher amount and proportion of terpenes. Floral scents dominated by terpenoids are common among plants pollinated by bees ${ }^{21}$, the main pollinator group during the early flowering period in this community (see Supplementary Fig. S1 online). Moreover, terpenoid emissions have been suggested to be major contributors to the effect of floral scent emissions on seed fitness ${ }^{16}$.

As expected based on the plant-abundance hypothesis, we found that Rosmarinus officinalis and Thymus vulgaris scent was different from that of less abundant co-flowering species, with the less abundant

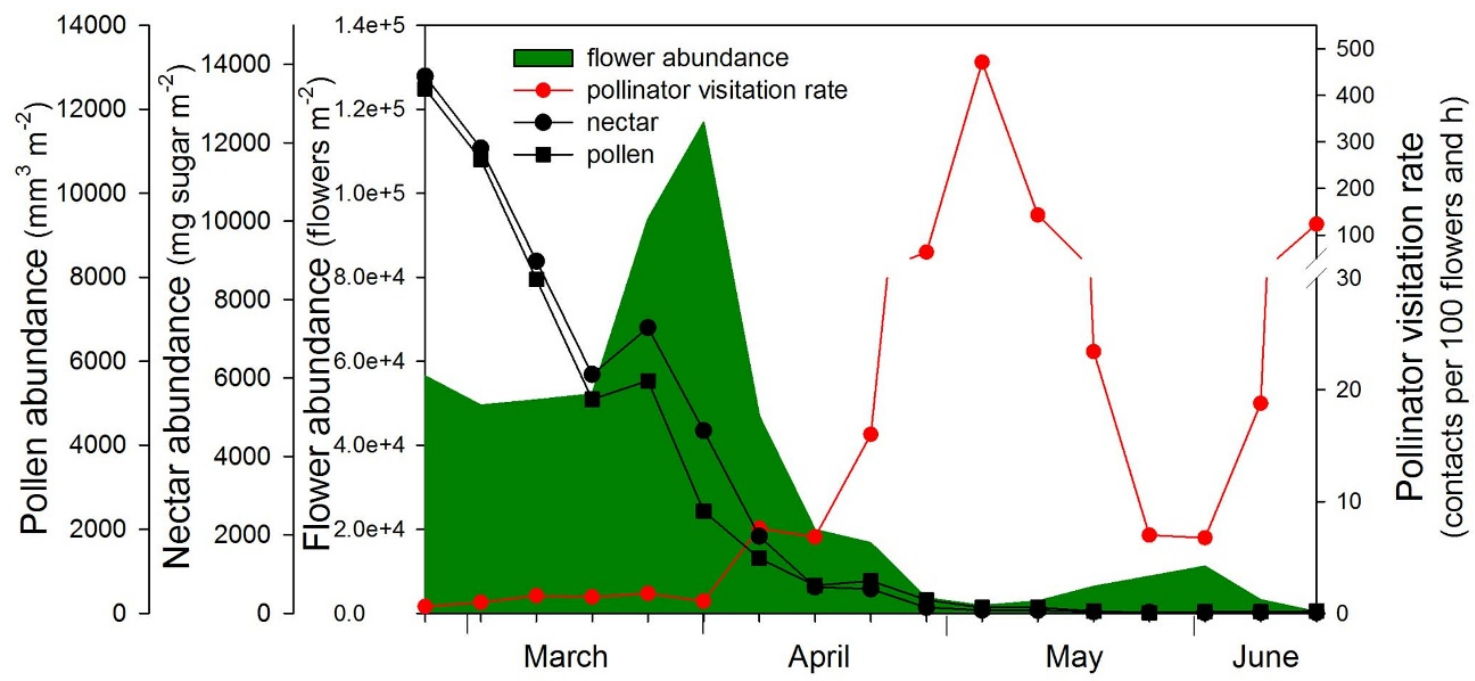

Figure 1 Seasonal pattern of weekly flower abundance (number of flowers per $\mathrm{m}^{2}$ ), floral rewards (nectar and pollen, $\mathrm{mg} \mathrm{sugar} \mathrm{and} \mathrm{mm}^{3}$ of pollen volume per $\mathrm{m}^{2}$ ), and pollinator visitation rate (number of pollinator contacts per 100 flowers and $\mathrm{h}$ ) in the Garraf plant-pollinator community in 2008. Note the break in the axis for the pollinator visitation rate. This seasonal pattern was consistent between years (authors' observations during the period 2006-2009). 


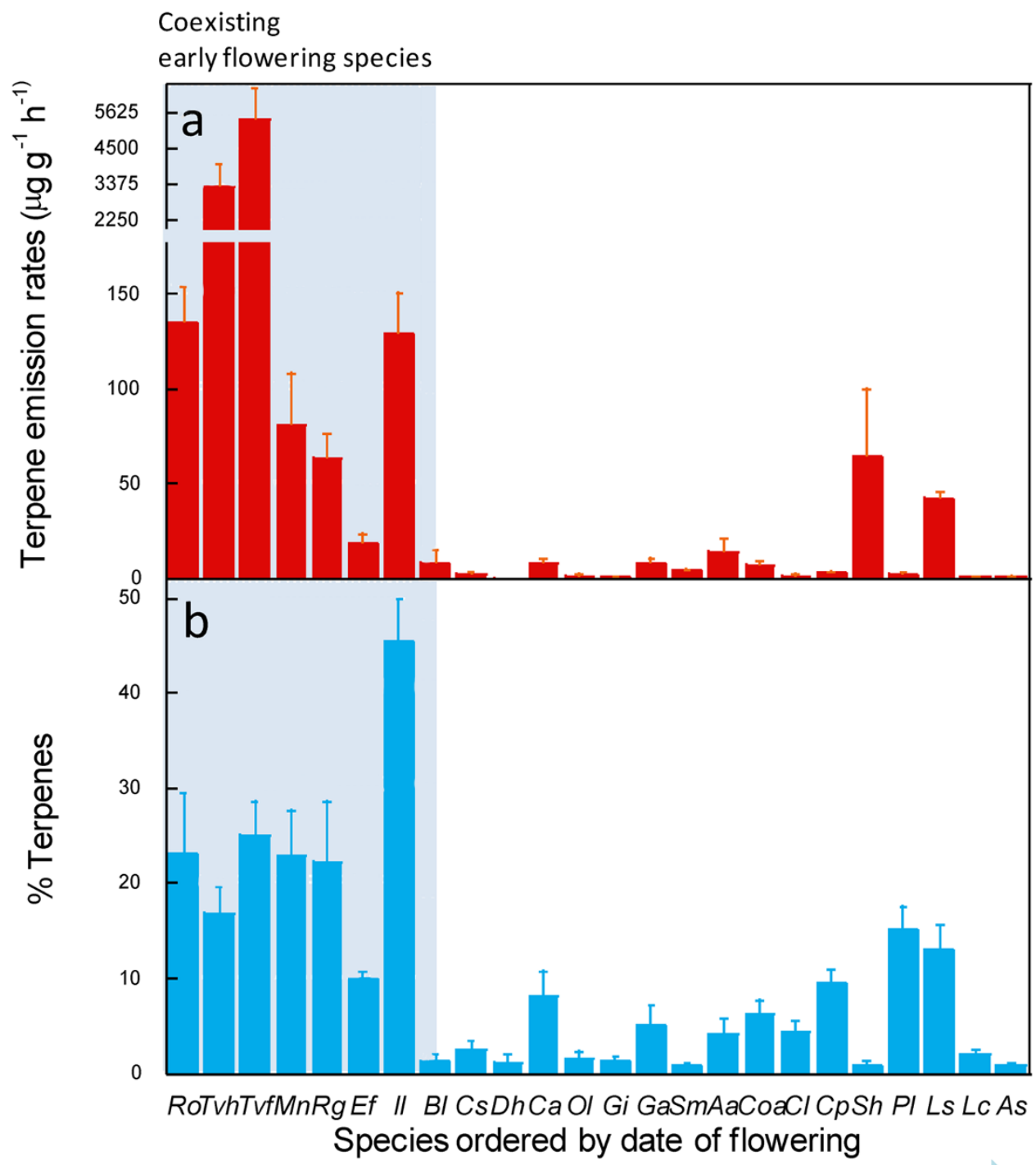

March

June

Figure $2 \mid$ Pollinator abundance hypothesis. Seasonal pattern of (a) terpene emission rates and (b) percentage of terpenes emitted relative to the total emission of biogenic volatile compounds by the plant species of the Garraf shrubland community ordered by date of flowering peak. Note the break in the axis for the terpene emission rates. Early (from late February to early April): Ro- Rosmarinus officinalis, Tvh-Thymus vulgaris hermaphrodite, Tvf- Thymus vulgaris female, Mn-Muscari neglectum, Rg-Ranunculus gramineus, Ef-Euphorbia flavicoma, Il-Iris lutescens; late (from early April to June): Cs- Cistus salvifolius, Dh-Dorycnium hirsutum, Ca-Cistus albidus, Ol-Orobanche latisquama, Gi-Gladiolus illyricus, Ga-Galium aparine, Sm-Scorpiurus muricatus, Aa- Anagallis arvensis, Coa-Convolvulus althaeoides, Cl- Centaurea linifolia, Cp-Centaurea paniculata, Sh-Sideritis hirsuta, Pl- Phlomis lychnitis, LsLinum strictum, Lc-Leuzea conifera, As-Allium sphaerocephalon. Bl-Biscutella laevigata blooms during most of the flowering season (March to June). Error bars are $\mathrm{SE}(\mathrm{n}=5)$.

species emitting a similar fragrance dominated by $\beta$-ocimene. $\beta$-ocimene is known to be a general attractant, emitted by a wide range of plants pollinated by different groups of pollinators ${ }^{22}$, such as bees ${ }^{23,24}$, moths ${ }^{25,26}$, butterflies ${ }^{27}$, and beetles ${ }^{26,28}$ and has been found to be attractive to honey bees and bumblebees ${ }^{29-31}$. Early flowering species shared pollinators, which for the most part were generalists ${ }^{20}$. Nondominant species would benefit from an increased capacity to attract pollinators and thus compensate for their low abundance. The existence of this shared long-range attraction odor does not prevent the existence of short-range differences among species that may lead to pollinator specialization. At least this seems to be the case in the genus Ranunculus, where it was found that $\beta$-ocimene presents an interesting spatial emission pattern within the flower with a marked increase in the emissions from the apical to basal part of the petals (nectariferous) paralleling optical nectar-guide patterns, and emission of protoanemonin associated exclusively with pollen and reproductive parts of the flowers ${ }^{32,33}$. While floral odours would operate at longer distances, the distinctiveness of the pollen's volatile profile suggests that it may serve a signaling role for pollinators specialized in collecting its pollen.

Variation in floral scent emission throughout the season could be the result of phenotypic plasticity ${ }^{34}$. The observed pattern could be attributed to a physiological flower response to pollinator abundance or to seasonal environmental changes (e.g., temperature, precipitation or air humidity). However, contrary to the expectations of a typical phenotypically plastic response, no significant seasonal trend was found in the studied species. Moreover, if anything, emissions would be expected to increase late in the season, when temperatures are higher and precipitation and air humidity lower ${ }^{35}$. The observed patterns could also be due to phylogenetic constraints, but there was 


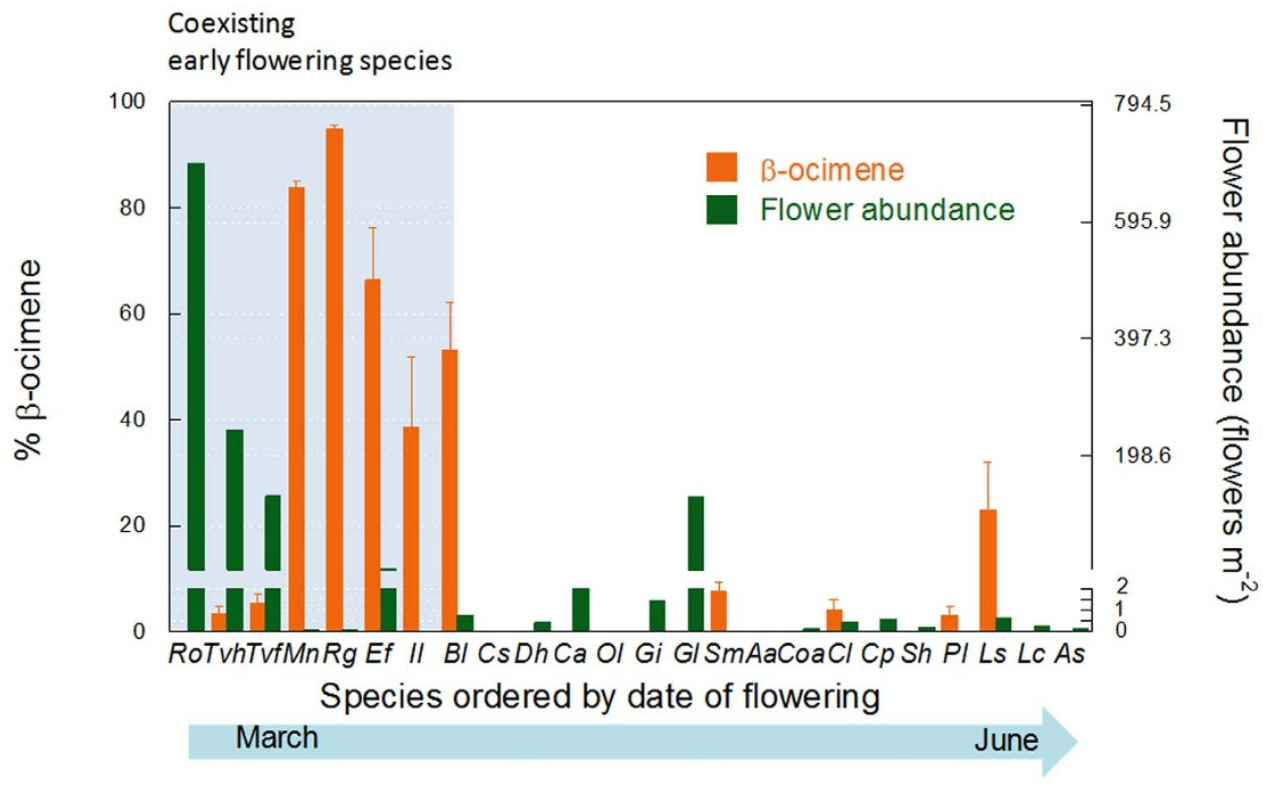

Figure 3 Plant abundance hypothesis. Flower abundance (number of flowers per $\mathrm{m}^{2}$ ) and percentage of $\beta$-ocimene emitted relative to the total emission of terpenes by the plant species of the Garraf shrubland community ordered by date of flowering peak as described in Figure 2. Note the break in the axis for the flower abundance. Note that although peaking in the second half of the season, Bl-Biscutella laevigata overlaps with the early flowering species throughout March and April. Error bars are SE $(n=5)$.

no significant phylogenetic signal for the total terpene emissions and the phylogenetic signal for $\beta$-ocimene percentage was not sufficient to explain the differences between the less abundant species on the one hand and R. officinalis and T. vulgaris on the other. Alternatively, the observed inter-specific differences in scent signals may be the result of adaptive processes. Parachnowitsch et al. ${ }^{36}$ found floral scent to be under stronger natural selection than either flower size or color, which are much more frequently examined in studies of floral evolution. Successful pollinator attraction and ultimate sexual reproduction in a plant species depend not only on the efficiency of its own scent signal but also on the efficiency of the signals of co-flowering species, in combination with their relative abundances, distribution and spatial intermixing. Thus, scent emission is likely to be under strong selective pressure conditioned by seasonal pollinator availability, and plant community species composition. The seasonal pattern could result from selection for high flower attractiveness under low pollinator availability. The scent pattern found in species

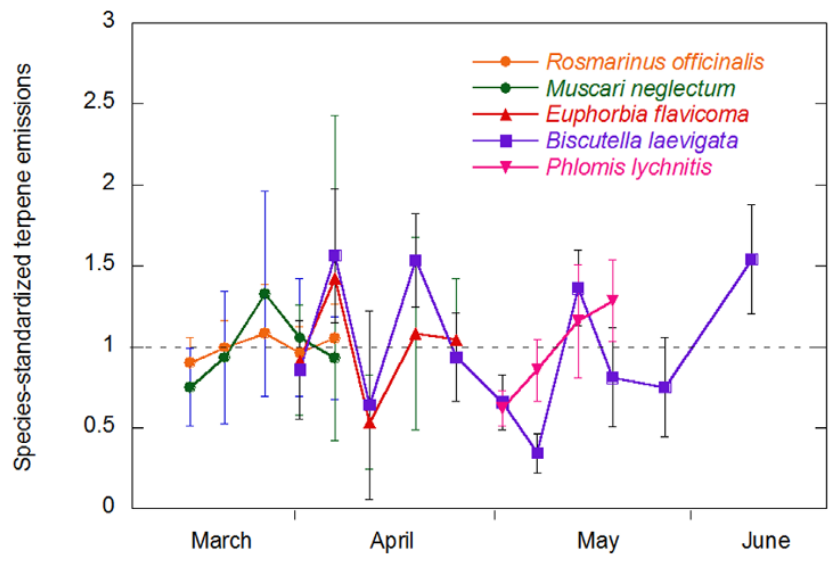

Figure $4 \mid$ Species-standardized terpene emission rates $\left(\mu \mathrm{g} \mathrm{g}^{-1} \mathbf{h}^{-1}\right)$ (emission rates were divided by the mean emission rate of each species) of five representative species throughout their entire blooming period. Error bars are $\mathrm{SE}(\mathrm{n}=5)$. Species standardized $\beta$-ocimene emission rates also followed no particular pattern (data not shown). co-flowering with the dominant $R$. officinalis and T. vulgaris may result from selection of those species with a scent detectable for pollinators even in the presence of the abundant scent of the dominant species.

With few exceptions ${ }^{37}$, studies analyzing the factors underlying the structure of plant-pollinator networks have mostly focused on abundance (neutrality models) and complementary phenological and morphological traits (trait matching models), while the potential contribution of volatiles has been largely ignored ${ }^{5}$. For the first time we show a clear divergent seasonal pattern of scent emission in a plant-pollinator community, with different levels of investment in scent advertisement, and unveil contrasting plant-signaling strategies associated with pollinator seasonal abundance and local plant abundance. Overall, we provide a first community-wide description of the seasonal dynamics of flower scent emissions, and report patterns that suggest a key role of flower scent signals in structuring plant-pollinator networks.

\section{Methods}

Study area field surveys. The study was conducted in a Mediterranean shrubland community in Garraf Natural Park (Barcelona, NE Spain), $340 \mathrm{~m}$ above sea level and $1700 \mathrm{~m}$ from the coastline. Field work was conducted in a ca. 1 ha plot, from late February to late June, encompassing the main flowering period in the area. No plants were in bloom during the dry summer season (July-August).

In 2008, we counted weekly the number of open flowers in six $50 \times 1 \mathrm{~m}$ transects and conducted pollinator counts on 24 plant species, representing $99.96 \%$ of the total number of flowers in the study plot: Rosmarinus officinalis, Thymus vulgaris (hermaphrodite and female morphs), Muscari neglectum, Ranunculus gramineus, Euphorbia flavicoma, Iris lutescens, Biscutella laevigata Cistus salvifolius, Dorycnium hirsutum, Cistus albidus, Orobanche latisquama, Gladiolus illyricus, Galium aparine, Scorpiurus muricatus, Anagallis arvensis, Convolvulus althaeoides, Centaurea linifolia, Centaurea paniculata, Sideritis hirsuta, Phlomis lychnitis, Linum strictum, Leuzea conifera and Allium sphaerocephalon. Floral rewards were measured on 15-20 flowers of each species. To measure volume of pollen produced per flower, we estimated the number of pollen grains in undehisced anthers in a 70\% ethanol-pollen suspending solution using an electronic particle counter (Coulter Multisizer), and measured pollen grain size under the microscope. To measure nectar production (mg of sugar produced per flower) we bagged flower buds and $24 \mathrm{~h}$ following anthesis, we used micropipettes to extract the accumulated nectar. Sugar concentration was measured with field refractometers.

Pollinator surveys were conducted twice a week throughout the blooming period. Flower patches were tagged, open flowers were counted and observed for $4 \mathrm{~min}$ periods throughout the day. During the observation time insects visiting the flowers 
were visually identified, and contacts were counted. Pollinators that could not be identified in the field were captured for later identification. From pollinator surveys, we obtained a measure of pollinator visitation rates (visits per flower and unit time).

Floral BVOC (biogenic volatile organic compounds) emission rates. We sampled the emission of flowers from 5 individuals of each plant species in its peak flowering week in 2009. Additionally, to test whether floral scent emission throughout the season could be the result of phenotypic plasticity, we sampled flowers from 5 individuals of 5 plant species ( $R$. officinalis, E. flavicoma, M. neglectum- species flowering mainly early in the season-, B. laevigata-flowering the whole season- and $P$. lychnitis-flowering late in the season) throughout their entire blooming period in 2011. In both cases samples were taken in the field at midday. We carefully put our specimens in water vials and immediately transferred them to a portable $4^{\circ} \mathrm{C}$ cabinet prior to analyses with gas chromatography (GC-MS) and Proton Transfer Reaction Mass Spectrometry (PTR-MS). BVOC analyses, with special focus on isoprenoids, were performed through head space technique in the GC-MS (Agilent Technologies, GC: 7890A, MS: 5975C inert MSD with Triple-Axis Detector, Palo Alto, CA, USA). In the laboratory, flowers (inflorescences in the case of Centaurea spp. and Leuzea conifera) were separated from vegetative parts. This procedure was applied for each of the 10 individuals of each plant species. Flowers were introduced in $10 \mathrm{ml}$ vials which were then placed in a Head Space incubator (CTC Analytics, MH 01-00B, Zwingen, Switzerland) and later processed with an automatic sample processor (Combi PAL, CTC Analytics, MXY 02-01B, Zwingen, Switzerland). Incubation time was $10 \mathrm{~min}$. at $35^{\circ} \mathrm{C}$. Two $\mathrm{ml}$ samples were injected into a $30 \mathrm{~m} \times 0.25 \mathrm{~mm} \times 0.25 \mu \mathrm{m}$ film thickness capillary column (HP-5MS, Agilent Technologies). Helium flow was $0.5 \mathrm{ml}$ $\mathrm{min}^{-1}$. Total run time was $30 \mathrm{~min}$. and the solvent delay was $4 \mathrm{~min}$. After the sample injection, the initial time was $1 \mathrm{~min}$. and the initial temperature $\left(40^{\circ} \mathrm{C}\right)$ was increased at $15^{\circ} \mathrm{C} . \mathrm{min}^{-1}$ up to $150^{\circ} \mathrm{C}$ and kept for $5 \mathrm{~min}$, and thereafter at $50^{\circ} \mathrm{C} \cdot \mathrm{min}^{-1}$ up to $250^{\circ} \mathrm{C}$ where the temperature was kept for $5 \mathrm{~min}$., and thereafter at $30^{\circ} \mathrm{C} \cdot \mathrm{min}^{-1}$ up to $280^{\circ} \mathrm{C}$, which was maintained for $5 \mathrm{~min}$. The identification of monoterpenes was conducted by comparing retention times with liquid standards from Fluka (Buchs, Switzerland) volatilized in the vial, and the fractionation mass spectra with standards spectra and Nist05a and wiley7n mass spectra libraries. Terpene concentrations were determined using calibration curves for common monoterpenes, alpha-pinene, betapinene, 3-carene, linalool, and sesquiterpene alpha-humulene. The analyses of emission rates for all emitted volatiles were conducted with a PTR-MS. Flowers were enclosed in a leaf cuvette of a LCpro + Photosynthesis System (ADC BioScientific Ltd., Hoddesdon, England) at $25^{\circ} \mathrm{C}$, and the air exiting the leaf cuvette was monitored with flow meters and analyzed with a Proton-Transfer-Reaction Mass Spectrometer (PTR-MS-FTD hs) from Ionicon Analytik, Innsbruck, Austria. These VOC analyses were replicated three times for each sample. The quantification of VOCs was based on the use of replicated three times calibration standards (ethylene, methanol, isoprene, $\alpha$-pinene, methyl salicylate and caryophylene, Sigma-Aldrich, Abelló- Linde). The PTR-MS drift tube was operated at $2.1 \mathrm{mbar}$ and $40^{\circ} \mathrm{C}$, with a drift field of $600 \mathrm{~V}$ $\mathrm{cm}^{-1}$. The parent ion signal was maintained at around $3 \times 10^{6}$ counts per second during the measurements. We conducted scans of all masses between 22 and 205 to determine which compounds were emitted by the different samples ${ }^{38}$. Previous to any measurement, we measured the background concentrations of VOCs in the empty cuvette, and considered these data to calculate the emission/uptake of every compound.

We estimated emission rates at the field temperature by using the equation

$$
\mathrm{M}=\mathrm{MTS} \exp (\mathrm{b}(\mathrm{T}-\mathrm{Ts}))^{39}
$$

where $\mathrm{M}$ is the emission rate at temperature T, MTS is emission rate at $303 \mathrm{~K}, \mathrm{~b}$ is an empirical coefficient and $\mathrm{Ts}=303 \mathrm{~K}$.

Data analysis. To test for differences between early and late-blooming plants in total BVOC emission, flower abundance, nectar and pollen content and pollinator abundance we conducted permutational multivariate ANOVAs (PERMANOVA) ${ }^{40}$ using the Bray Curtis index of similarity, with "season" (early flowering period, late flowering period) as a fixed factor. We also conducted a cluster analysis on the percentage of the different VOCs emitted by each species. All these analyses were conducted using the statistical packages PERMANOVA+ for PRIMER v. $6^{40}$ and Statistica 6.0 (Statsoft Inc., Tulsa, OK, USA). We also used the program PHYLOMATIC ${ }^{41}$ to build a phylogenetic tree of the plant species studied and test if total terpene emission showed a significant phylogenetic signal- i.e. the tendency of closely related species to resemble each other due to shared ancestry- as described $\mathrm{in}^{42}$. Briefly, PHYLOMATIC uses a backbone plant megatree based on a variety of sources involving primarily DNA studies to assemble a phylogenetic tree for the species of interest. Our phylogenetic hypothesis was based on the conservative megatree, where unresolved nodes were included as soft polytomies. We used the PDAP package ${ }^{43}$ to transform the phylogenetic tree into a matrix of phylogenetic distances, and tested if the studied traits showed significant phylogenetic signal with the randomization procedure in the PHYSIG module developed by ${ }^{45}$. This test compares the variance in phylogenetic independent contrasts observed in the real dataset against a null distribution obtained when the phenotypic data are randomized across the tips of the tree (breaking any pattern of phylogenetic resemblance between relatives). Phylogenetic signal was considered significant if the variance in contrasts of the real dataset was lower than the variance in $95 \%$ of the permuted datasets. These analyses were performed to determine if phylogenetic correction was necessary in subsequent regression analyses. When the dependent variable showed significant phylogenetic signal we used phylogenetic generalized least square regressions (PGLS). PGLS controls for phylogenetic relatedness by adjusting the expected variance/covariance of regression residuals using the matrix of phylogenetic distances (this approach is mathematically equivalent to analyzing the data with phylogenetically independent contrasts). These analyses were performed with the REGRESSIONV2 module in MATLAB 7.6. $0^{46}$. We used the stats package ${ }^{44}$ to draw the heatmap of volatile emissions in each species.

1. Cohen, D. \& Shmida, A. The evolution of flower display and reward. Evol. Biol. 27, 197-243 (1993).

2. Chittka, L. \& Schürkens, S. Successful invasion of a floral market. Nature 41 1, 653 (2001).

3. Noe, R. \& Hammerstein, P. Biological markets. Trends Ecol. Evol. 10, 336-339 (1995).

4. Chittka, L. \& Raine, N. E. Recognition of flowers by pollinators. Curr. Opin. Plant Biol. 9, 428-435 (2006).

5. Raguso, R. A. Start making scents: the challenge of integrating chemistry into pollination ecology. Entomol. Exp. Appl. 128, 196-207 (2008).

6. Wright, G. \& Schiestl, F. P. The evolution of floral scent: the influence of olfactory learning by insect pollinators on the honest signalling of floral rewards. Funct. Ecol. 23, 841-851 (2009).

7. Dobson, H. E. M. Role of flower and pollen aromas in host plant recognition by solitary bees. Oecologia 72, 618-623 (1987).

8. Dobson, H. E. M. \& Bergström, G. The ecology of pollen odor. Plant. Syst. Evol. 222, 63-87 (2000).

9. Goulson, D., Chapman, J. W. \& Hughes, W. O. H. Discrimination of unrewarding flowers by bees; direct detection of rewards and use of repellent scent marks. J. Insect Behav. 14, 669-678 (2001).

10. Howell, A. D. \& Alarcon, R. Osmia bees (Hymenoptera: Megachilidae) can detect nectar-rewarding flowers using olfactory cues. Anim. Behav. 74, 199-205 (2007).

11. Menzel, R. in Neurobiology of learning and memory in honeybees. The Behaviour and Physiology of Bees (eds Goodman, L. J. \& Fisher, R. C.) 323-354 (CAB International, Wallingford, 1991).

12. Waelti, M. O., Muhlemann, J. K., Widmer, A. \& Schiestl, F. P. Floral odour and reproductive isolation in two species of Silene. J. Evol. Biol. 21, 111-121 (2008).

13. Cook, S. M., Bartlet, E., Murray, D. A. \& Williams, I. H. The role of pollen odour in the attraction of pollen beetles to oilseed rape flowers. Entomol. Exp. Appl. 104, 43-50 (2002).

14. Anderson, S. Foraging responses in the butterflies Inachis io, Aglais urticae (Nymphalidae), and Gonopteryx rhamni (Pieridae) to floral scents. Chemoecol. 13, 1-11 (2003).

15. Primante, C. \& Dötterl, S. A syrphid fly uses olfactory cues to find a non-yellow flower. J. Chem. Ecol. 36, 1207-1210 (2010).

16. Majetic, C. J., Raguso, R. A. \& Ashman, T. L. The sweet smell of success: floral scent affects pollinator attraction and seed fitness in Hesperis matronalis. Funct. Ecol. 23, 480-487 (2009).

17. Kummerov, J. Comparative phenology of Mediterranean type plant communities. In Mediterranean plant ecosystem, (eds Kruger, F. J., Mitchell, D. L. \& Jarvis, J. U. M.) 300-317 (Springer Verlag, Berlin, 1983).

18. Bosch, J., Retana, J. \& Cerdá, X. Flowering phenology, floral traits and pollinator composition in a herbaceous Mediterranean plant community. Oecologia 109, 583-591 (1997).

19. Petanidou, T., Ellis, W. N., Margaris, N. S. \& Vokou, D. Constraints on flowering phenology in a phryganic (east Mediterranean shrub) community. Am. J. Bot. 82, 607-620 (1995)

20. Bosch, J., Martín González, A. M., Rodrigo, A. \& Navarro, D. Plant-pollinator networks: adding the pollinator's perspective. Ecol. Lett. 12, 409-419 (2009).

21. Dobson, H. E. M. in Biology of Floral Scent (eds Dudareva, N. \& Pichersky, E.) 147-198 (Taylor and Francis, Boca Raton, 2006).

22. Knudsen, J. T., Eriksson, R., Gershenzon, J. \& Stahl, B. Diversity and distribution of floral scent. Bot. Rev. 72, 1-120 (2006).

23. Gerlach, G. \& Schill, R. Composition of orchid scents attracting euglossine bees. Bot. Acta 104, 379-391 (1991).

24. Borg-Karlson, A., Valterova, I. \& Nilsson, L. A. Volatile compounds from flowers of six species in the family Apiaceae: bouquets for different pollinators? Phytochem. 35, 111-119 (1994).

25. Knudsen, J. T. \& Tollsten, L. Trends in floral scent chemistry in pollination syndromes: floral scent composition in moth-pollinated taxa. Bot. J. Linn. Soc. 113, 263-284 (1993).

26. Okamoto, T., Kawakita, A. \& Kato, M. Interspecific variation of floral scent composition in Glochidion and its association with host-specific pollinating seed parasite (Epicephala). J. Chem. Ecol. 33, 1065-1081 (2007).

27. Andersson, S., Nilsson, L. A., Groth, I. \& Bergström, G. Floral scents in butterflypollinated plants: possible convergence in chemical composition. Bot. J. Linn. Soc. 140, 129-153 (2002).

28. Dufaÿ, M., Hossaert-McKey, M. \& Anstett, M. C. When leaves act like flowers; how dwarf palms attract their pollinators. Ecol. Lett. 6, 28-34 (2003).

29. Loper, G. M., Waller, G. D. \& Berdel, R. L. Olfactory screening of alfalfa clones for uniform honeybee selection. Crop Sci. 14, 120-122 (1974). 
30. Pecetti, L., Tava, A., Felicioli, A., Pinzauti, M. \& Piano, E. Effect of three volatile compounds from lucerne flowers on their attractiveness towards pollinators. B. Insectol. 55, 21-27 (2002).

31. Granero, A. M. et al. Chemical compounds of the foraging recruitment pheromone in bumblebees. Naturwissenschaften 92, 371-374 (2005)

32. Bergström, G., Dobson, H. E. M. \& Groth, I. Spatial fragrance patterns within the flowers of Ranunculus acris (Ranunculaceae). Plant Syst. Evol. 195, 221-242 (1995).

33. Jürgens, A. \& Dötterl, S. Chemical composition of anther volatiles in Ranunculaceae: Genera-specific profiles in Anemone, Aquilegia, Caltha, Pulsatilla, Ranunculus, and Trollius species. Am. J. Bot. 91, 1969-1980 (2004)

34. Majetic, C. J., Raguso, R. A. \& Ashman, T. L. Sources of floral scent variation. Can environment define floral scent phenotype? Plant. Signal Behav. 4, 129-131 (2009)

35. Jakobsen, H. B. \& Olsen, C. E. Influence of climatic factors on emission of flower volatiles in situ. Planta 192, 365-371 (1994).

36. Parachnowitsch, A. L., Raguso, R. A. \& Kessler, A. Phenotypic selection to increase floral scent emission, but not flower size or colour in bee-pollinated Penstemon digitalis. New Phytol. 195, 667-675 (2012).

37. Junker, R. R., Höcherl, N. \& Blüthgen, N. Responses to olfactory signals reflect network structure of flower-visitor interactions. J. Anim. Ecol. 79, 818-23 (2010).

38. Peñuelas, J., Filella, I., Stefanescu, C. \& Llusià, J. Caterpillars of Euphydryas aurinia (Lepidoptera: Nymphalidae) feeding on Succisa pratensis leaves induce large foliar emissions of methanol. New Phytol. 167, 851-857 (2005).

39. Guenther, A., Zimmerman, P., Harley, P., Monson, R. \& Fall, R. Isoprene and monoterpene emission rate variability: model evaluation and sensitivity analysis. J. Geophys. Res. 98, 12609-12617 (1993).

40. Anderson, M. J., Gorley, R. N. \& Clarke, K. R. PERMANOVA+ for PRIMER Guide to Software and Statistical Methods. Plymouth: PRIMER-E. 214 pp (2008).

41. Webb, C. O. \& Donoghue, M. J. Phylomatic: tree assembly for applied phylogenetics. Mol. Ecol. Notes 5, 181-183 (2005).

42. Peñuelas, J. et al. Faster returns on "leaf economics" and different biogeochemical niche in invasive compared with native plant species. Global Change Biol. 16, 2171-2185 (2010)

43. Garland, T. J., Harvey, P. H. \& Ives, A. R. Procedures for the analysis of comparative data using phylogenetically independent contrast. Syst. Biol. 41, 18-32 (1993).
44. R Core Team. R: A language and environment for statistical computing. $R$ Foundation for Statistical Computing, Vienna, Austria. (2013).

45. Blomberg, S. P., Garland, T. J. \& Ives, A. R. Testing for phylogenetic signal in comparative data: behavioral traits are more labile. Evolution 57, 717-745 (2003).

46. Lavin, S. R., Karasov, W. H., Ives, A. R., Middleton, K. M. \& Garland, T. J. Morphometrics of the avian small intestine compared with that of nonflying mammals: a phylogenetic approach. Physiol. Biochem. Zool. 81, 526-550 (2008).

\section{Acknowledgments}

This research was supported by funding from Spanish Government grants CGL2005-00491, CGL2009-12646, CGL2010-17172/BOS and Consolider-Ingenio Montes (CSD2008-0040) and from the Catalan Government grant CSGR2009/458. We thank J. Piñol for assistance with PERMANOVA analyses.

\section{Author contributions}

I.F., A.R., J.B. and J.P. designed the research. I.F., C.P., A.M.G., G.F., A.R. and J.B. conducted the field work. I.F., J.L., R.S., G.F. and J.P. conducted the lab analyses. I.F. and J.P. drafted the paper. All authors contributed to the interpretation of the results and were deeply involved in the writing of the text.

\section{Additional information}

Supplementary information accompanies this paper at http://www.nature.com/ scientificreports

Competing financial interests: The authors declare no competing financial

How to cite this article: Filella, I. et al. Floral advertisement scent in a changing plant-pollinators market. Sci. Rep. 3, 3434; DOI:10.1038/srep03434 (2013).

(i) $\Theta$ This work is licensed under a Creative Commons AttributionBY NC ND NonCommercial-NoDerivs 3.0 Unported license. To view a copy of this license visit http://creativecommons.org/licenses/by-nc-nd/3.0 